\title{
Trabajo y rendimiento académico en estudiantes de Educación Básica Alternativa altoandina
}

\author{
Work and academic performance in students of high \\ andean alternative basic education
}

\author{
Flor María Arauco Acosta 1,a \\ https://orcid.org/0000-0002-5919-3236 \\ Jorge Constantino Arauco López 2,b \\ https://orcid.org/0000-0001-6676-1193 \\ Cecilia Gladys Aliaga Sandoval 2,c \\ https://orcid.org/0000-0003-3470-1895
}

\section{Citar como}

Arauco Acosta F, Arauco López J, y Aliaga Sandoval C. (2020). Trabajo y rendimiento académico en estudiantes de educación básica alternativa altoandina. Desafíos, 2020; 17(1); 32-8. doi: https://doi.org/10.37711/desafios.2020.11.1.139

\section{RESUMEN}

Objetivo. Describir las características del trabajo en el rendimiento académico de los estudiantes del CEBA María Inmaculada. Métodos. Estudio de tipo básico, nivel descriptivo y técnicas mixtas, diseño de investigación: explicativo secuencial (DEXPLIS). La muestra estuvo constituida por 31 estudiantes que trabajan de 14 a 18 años. La metodología estadística usada fue descriptiva y se emplearon pruebas de chi cuadrado. Resultados. Se encontró que las características laborales de los estudiantes son muy duras en su mayoría, resaltando horarios laborales superiores a 8 horas diarias, trabajos diurnos de subsistencia. En relación al rendimiento académico se reportó que el 64 \% presentó calificaciones admisibles de logros, previsto según la escala nacional de aprendizaje (ECA) normada por el Ministerio de Educación del Perú; ajustada a las escalas literal, descriptiva y categórica. El resto de estudiantes está en proceso de aprendizaje, pero ninguno con deficiencias o reprobación académica. También se determinó que la legitimidad del contrato, la jornada de trabajo, la retribución mensual, el periodo de pago, la autorización de los padres para trabajar y la autonomía del trabajo no se relacionan significativamente con el rendimiento académico ( $p>0,05)$. Conclusión. A pesar de tener condiciones de trabajo muy básicas y defectuosas con faltas graves a las políticas laborales, los resultados muestran que los estudiantes tienen buen desempeño académico; denotando por ello una capacidad de autosuperación inmutable enriquecedora para su formación, pero conminándonos a proteger sus derechos mediante normas y condiciones que regulen el trabajo adolescente por parte de los gobiernos locales y nacionales.

Palabras clave: trabajo adolescente, rendimiento escolar, características laborales, logros de aprendizaje, Educación Básica Alternativa.

\section{ABSTRACT}

Objective. To describe the characteristics of work in the academic performance of students from Educational Center of Basic Education (CEBA) María Inmaculada. Methods. Basic type study, descriptive level and mixed techniques, research design: sequential explanatory (DEXPLIS). The sample comprised of 31 students that work from 14 to 18 years old. The statistical methodology used was descriptive and chi square tests were used. Results. It was found that the labor characteristics of the students are mostly very harsh, highlighting working hours greater than 8 hours a day, daytime subsistence jobs. Regarding academic performance, it was reported that $64 \%$ presented admissible grades of achievement, predicted according to the national learning scale (ECA) regulated by the Department of Education of Peru. It is adjusted to the literal, descriptive and categorical scales. The rest of the students are in a

\section{Filiación y grado académico}

1 Investigador en la Universidad Nacional del Centro Perú.

2 Universidad Nacional del Centro Perú. Huancayo, Perú.

\author{
a Licenciada en Trabajo Social. \\ b Magíster en Didáctica Universitaria \\ c Magíster en Políticas Sociales.
}


learning process, but none with academic deficiencies or failed grades. It was also determined that the legitimacy of the contract, the working days, the monthly salary, the payment period, the parental authorization to work and the autonomy of the work are not significantly related to academic performance ( $p>0.05)$. Conclusion. Despite having very basic and defective working conditions with serious violations of labor policies, the results show that students have good academic performance. In addition, they showed an immutable self-improvement capacity that improves their training. However, it is important to protect their rights through norms and conditions that regulate adolescent work by local and national governments.

Keywords: adolescent work, school performance, work characteristics, learning achievements, Alternative Basic Education.

\section{INTRODUCCIÓN}

El estudio presenta resultados sobre la relación causal que existe entre las características del trabajo adolescente y el rendimiento escolar, pues se considera que el trabajo adolescente es un problema económico en tanto que reduce la acumulación de capital humano del menor y afecta su productividad e ingresos futuros (OIT, 2002; UNICEF, 2010). En la última década se reportó que el 33\% de los adolescentes entre 12 y 18 años realiza actividades económicas y la mitad de ellos combina el trabajo con el estudio. Se estima que, en el ámbito rural, el $40 \%$ de niños y adolescentes en el Perú trabaja en actividades agropecuarias y mineras con serios efectos en la escolaridad; mientras que el $49 \%$ de los que trabajan y asisten a la escuela tiene uno o más años de atraso escolar (UNICEF, 2016). Los niños en áreas altoandinas que provienen de familias pobres tienen un nivel educativo más bajo, puntajes más bajos en los exámenes, menor asistencia escolar y menos años de escolaridad, que los niños nacidos en áreas urbanas o en una familia rica (Chinvoka y Naidu, 2014). La explicación de esto es que los niños rurales necesitan participar en más actividades laborales que los niños urbanos.

Debido a la problemática de adolescentes que trabajan y estudian, se crearon los Centros de Educación Básica Alternativa (CEBA), a fin de no sustraer el derecho a la educación de esta población vulnerable, como se puede leer que en la Resolución Viceministerial N. ${ }^{\circ}$ 025-2019 del Ministerio de Educación del Perú (MINEDU, 2019). Los CEBA fueron creados bajo la Ley General de Educación N.o. 28044, enfatizando la preparación para el trabajo y el desarrollo de capacidades empresariales de los estudiantes adolescentes, jóvenes y adultos, afectados por diversas cuestiones como problemas familiares, escasos recursos económicos, embarazo adolescente, entre otros (Vilcarana y Cayllahua, 2017). Los estudiantes altoandinos son más vulnerables y tienen serias dificultades para matricularse en el centro educativo, debido a que muchos de ellos se desligan de la Educación Básica Regular, sobrellevando una serie de trabas para su reincorporación; lo que perjudica su incorporación inmediata al mundo escolar. A esto se suma el hecho de que tienen que trabajar a diario para la subsistencia de sus familias (Ovalle, 2009); provocando que su asistencia sea irregular en el centro educativo.

Mediante la problemática presentada, la muestra es pequeña pero significativa, por representar a una población en situación de exclusión. Su estudio nos permite plantear propuestas para mejorar su situación laboral y rendimiento académico, enmarcándolo en el bienestar social del adolescente y buscando el equilibrio entre la función positiva del trabajo y la vida de los adolescentes. En ese sentido, el estudio explica la influencia de las características del trabajo adolescente en el rendimiento académico.

\section{MÉTODOS}

\section{Tipo de estudio}

El método de investigación fue el descriptivo y el enfoque mixto. El estudio se desarrolló con 31 adolescentes que trabajan, cuyas edades fluctúan entre 14 a 18 años y estudian en el Centro Educativo Básico Alternativo (CEBA) "María Inmaculada", de la Ciudad de Huancayo - Perú.

\section{Instrumentos de medición}

Se elaboró y aplicó un cuestionario para recoger datos cuantitativos y una guía de entrevista de 11 preguntas para recoger datos cualitativos verificando su confiabilidad y validez mediante juicio de expertos y pruebas piloto a través del método de Lawshe (Lawshe, 1975). Adicionalmente se revisó el registro de récord académico y se cotejó la Escala de Calificación de Aprendizaje (ECA) a través del empleo de los promedios ponderados. Para analizar el rendimiento académico, los promedios ponderados fueron transformados en 
base al ECA, en la Educación Básica Regular (ver tabla 1), normado por el Ministerio de Educación del Perú (MINEDU) por Resolución Viceministerial N025-2019 (p. 55); mientras que la escala numérica de 0 a 20 fue ajustada a la escala literal y descriptiva.

\section{Análisis de datos}

Los datos se analizaron con tablas cruzadas y análisis descriptivo, con técnicas e instrumentos de contraste de hipótesis, mediante la prueba de chi cuadrado. Prueba exacta de Fisher a un nivel de confianza del $95 \%(p<0,05)$ para analizar la relación entre variables cualitativas y homogeneidad de muestras en el software STATAGRAPHICS centurión.

\section{RESULTADOS}

Los porcentajes obtenidos de la caracterización del lugar y tipo de trabajo de los estudiantes evaluados en el CEBA María Inmaculada se muestran en la figura 1, donde se encontró que la mayor proporción de los alumnos que trabajan lo hacen en negocios, talleres, locales, fábricas (envasando néctares), representando al 74,2 \% del total. A continuación, se encuentran los alumnos que laboral en la calle como ambulante o en el rubro de transporte urbano, como cobradores, con un $12,9 \%$ del total. El último grupo en importancia proporcional lo constituyen las alumnas que trabajan como servidoras del hogar o como ayudantes de limpieza o ayudan con los quehaceres de las casas donde son contratadas, quienes representan el 9,7\% del total. Asimismo, los tipos de trabajo que realizan los alumnos son múltiples. En tal sentido, existen aquellos que se dedican al comercio, principalmente como negociantes, principalmente como negociante, siendo el grupo más representativo con el 22,6\% del total. Este grupo es seguido por aquellos varones principalmente,
Tabla 1

Equivalencias de escalas de calificación según el Ministerio de Educación (MINEDU) para Educación Básica Regular

\begin{tabular}{lc}
\hline Escala de calificación literal & $\begin{array}{c}\text { Escala de calificación } \\
\text { numérica }\end{array}$ \\
\hline Logro destacado & $20-18$ \\
Logro previsto & $17-14$ \\
En proceso & $13-11$ \\
En inicio & $10-00$ \\
\hline
\end{tabular}

Nota: (MINEDU, 2019).

que trabajan como ayudantes en construcción (generalmente civil), quienes representan el 19,4\% del total de alumnos encuestados. El tercer grupo representativo está conformado por alumnos que trabajan como camareros (mozos) en restaurantes, tendiendo a encontrar unidades muéstrales de ambos sexos en este rubro. Este grupo constituye el 16,1 \% de la muestra; además, para el rubro de los restaurantes se encuentra que, del total de encuestados el 9,68 \% trabaja en cocina con la manipulación de los alimentos, siendo el sexo femenino el más representativo en este conjunto.

Las proporciones obtenidas para la caracterización del horario, horas de trabajo, ingresos y destino de gastos de los estudiantes evaluados en el CEBA se detallan en la figura 2. Según los horarios de trabajo se encontró que la proporción más importante es del 83,9 \% con característica de trabajo matutino. El resto de grupos, con una menor representatividad, trabaja durante las madrugadas, mañana-tarde. Las horas de trabajo muestran que el 41,9\% labora por 10 horas diarias, seguido por el $35,5 \%$ que labora 8 horas diarias aproximadamente; siendo estos grupos representativos para este factor. Los grupos menos significativos los conforman alumnos que laboran entre 6 y 4 horas, con proporciones del 12 y $9 \%$ respectivamente. Respecto al periodo de pago al 45,2 \%
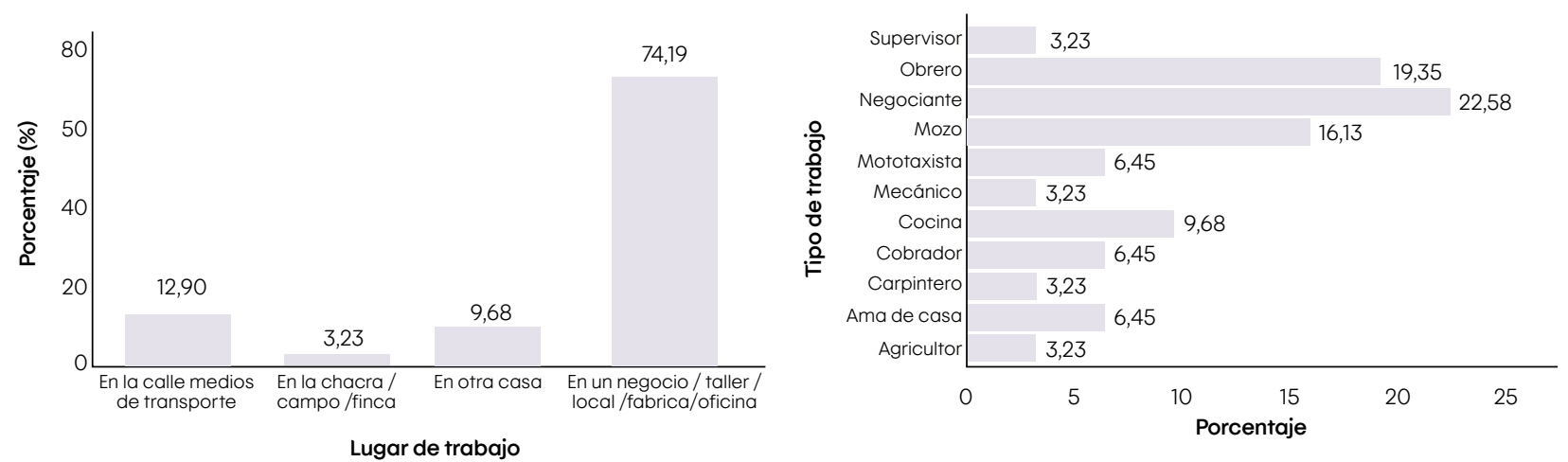

Figura 1. Frecuencia porcentual de lugares y tipo de trabajo de estudiantes de Educación Básica Alternativa del CEBA María Inmaculada de Huancayo. 

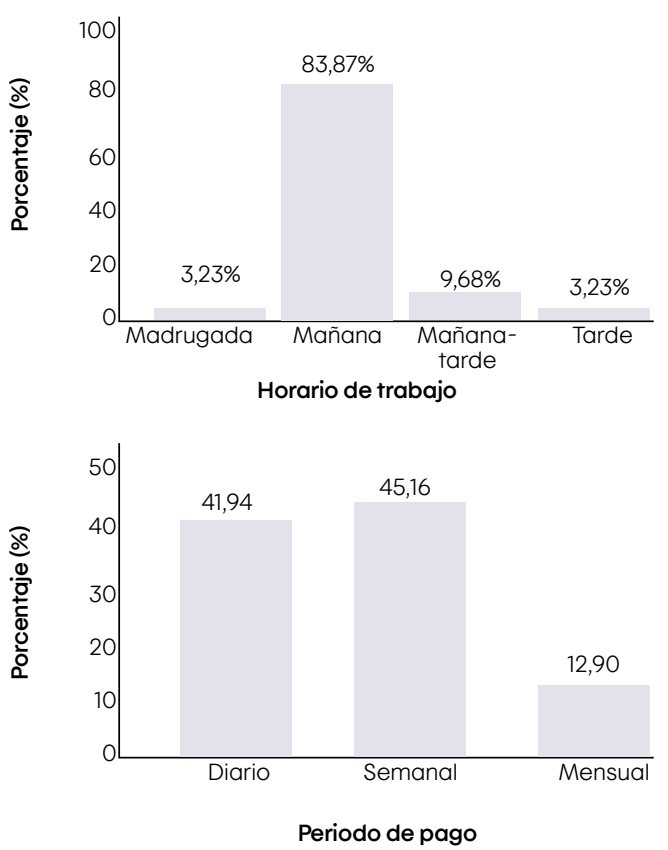
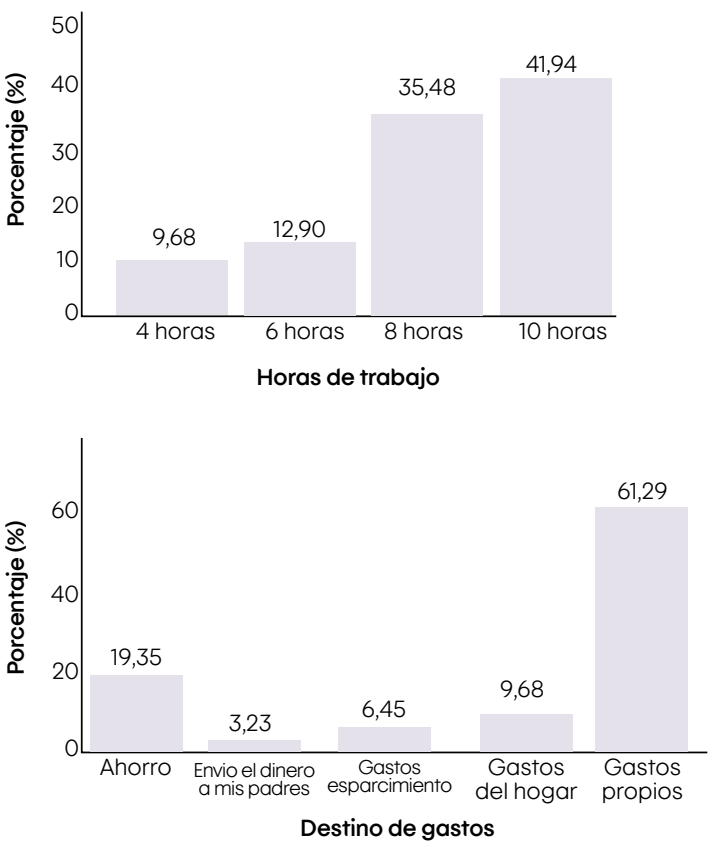

Figura 2. Frecuencia porcentual de horarios de trabajado, peculiaridades de pago y gastos de estudiantes de Educación Básica Alternativa que laboran del CEBA María Inmaculada de Huancayo.

de los alumnos les pagan semanalmente (obreros y mozos); mientras que el $41,9 \%$ percibe ingresos diarios, siendo estos en su mayoría independientes, (por lo general, comerciantes ambulatorios). En función a los gastos se reportó que el $61,3 \%$ lo emplean principalmente para la alimentación, bienestar, materiales educativos y salud, siendo este grupo el más representativo; seguido por aquellos que ahorran los ingresos con un $19,4 \%$ de representatividad.

De acuerdo a los resultados que muestra la tabla 2 con respecto a la autonomía del trabajo, el trabajo en su mayoría $(64,5 \%)$ indica que son

\section{Tabla 2}

Particularidades laborales de los estudiantes de Educación Básica Alternativa del CEBA María Inmaculada de Huancayo

\begin{tabular}{|c|c|c|c|c|}
\hline \multirow{3}{*}{$\begin{array}{l}\text { Característica } \\
\text { Autonomía }\end{array}$} & \multicolumn{4}{|c|}{ Frecuencias relativas por nivel } \\
\hline & \multicolumn{2}{|c|}{ Dependiente } & \multicolumn{2}{|c|}{ Independiente } \\
\hline & $\mathrm{fi}$ & $\%$ & $\mathrm{fi}$ & $\%$ \\
\hline & 11 & 64,5 & 20 & 35,5 \\
\hline \multirow{2}{*}{ Legitimidad del contrato } & \multicolumn{2}{|c|}{ Formal } & \multicolumn{2}{|c|}{ Informal } \\
\hline & 8 & 25,8 & 23 & 74,2 \\
\hline \multirow{2}{*}{$\begin{array}{l}\text { Autorización de } \\
\text { apoderados para trabajar }\end{array}$} & \multicolumn{2}{|c|}{$\mathrm{Si}$} & \multicolumn{2}{|c|}{ No } \\
\hline & 27 & 87,1 & 4 & 12,9 \\
\hline \multirow[t]{2}{*}{ Remuneración } & \multicolumn{2}{|c|}{$\begin{array}{l}\text { Menor o } \\
\text { igual } 800\end{array}$} & \multicolumn{2}{|c|}{ Mayor 800} \\
\hline & 12 & 38,7 & 19 & 61,3 \\
\hline \multirow[t]{2}{*}{ Forma de pago } & \multicolumn{2}{|c|}{ Efectivo } & \multicolumn{2}{|c|}{$\begin{array}{c}\text { Especies o productos } \\
\text { perecibles }\end{array}$} \\
\hline & 26 & 83,9 & 5 & 16,1 \\
\hline
\end{tabular}

Nota: Instrumento de evaluación elaborado por autores. trabajadores dependientes; considerando que están bajo condiciones de subordinación.Asimismo, se observa que la mayoría (74,2\%) se encuentra laborando de manera informal. Además, aquellos que cuentan con autorización de sus progenitores representan al $87,1 \%$ del total; mientras que el $61,3 \%$ percibe un sueldo mayor a los 800 soles y el $83,9 \%$ recibe su pago en efectivo.

Los resultados de los promedios en la escala de calificación numérica de los alumnos fueron de 14,25 \pm 0,96 con mínimo de 12 y máximo de 17. El $50 \%$ de los datos, aproximadamente la media, están entre los valores de 14 y 15. Se encontró que el $64,5 \%$ de la muestra de alumnos que trabajan tiene un valor de logro previsto en su rendimiento académico, según el ECA categórico, y el 35,5\% de los alumnos que trabajan muestreados, que trabajan tuvo un valor del proceso de aprendizaje, ya que sus notas oscilaron entre 11 y 13.

La tabla 4 muestra con qué frecuencia se presentan los 2 valores de ECA junto con cada uno de los valores de las características laborales. El primer número de cada celda en la tabla es el recuento o frecuencia y el segundo número muestra el porcentaje de toda la tabla que

\section{Tabla 3}

Frecuencia de rendimiento académico según escala de calificación de aprendizaje (ECA) de alumnos

\begin{tabular}{ccc}
\hline Valor & fi & $\%$ \\
\hline En proceso & 11 & 35,48 \\
Logro previsto & 20 & 64,52 \\
\hline Nota: Elaborado por autores, considerando los criterios del MINEDU.
\end{tabular}


Tabla 4

Tabla de contingencia para ECA según características laborales de los estudiantes de Educación Básica Alternativa del CEBA María Inmaculada de Huancayo

\begin{tabular}{|c|c|c|c|c|c|c|}
\hline \multirow{3}{*}{ Características del trabc } & & \multicolumn{4}{|c|}{ Nivel de logro de aprendizaje } & \multirow{3}{*}{ Valor - $p$} \\
\hline & & \multicolumn{2}{|c|}{ En proceso } & \multicolumn{2}{|c|}{ Logro previsto } & \\
\hline & & $\mathrm{fi}$ & $\%$ & $\mathrm{fi}$ & $\%$ & \\
\hline \multirow{2}{*}{ Tipo } & Formal & 2 & 6,45 & 6 & 19,35 & \multirow{2}{*}{0,6757} \\
\hline & Informal & 9 & 29,03 & 14 & 45,16 & \\
\hline \multirow{2}{*}{ Jornada } & Menor o igual a 48 horas semanales & 6 & 19,35 & 16 & 51,61 & \multirow{2}{*}{0,2175} \\
\hline & Superior a 48 horas semanales & 5 & 16,13 & 4 & 12,9 & \\
\hline \multirow{2}{*}{ Retribución mensual } & Mayor a 930 & 1 & 3,23 & 11 & 35,48 & \multirow{2}{*}{0,0201} \\
\hline & Menor o igual a 930 & 10 & 32,26 & 9 & 29,03 & \\
\hline \multirow{3}{*}{ Periodo de pago } & Diario & 6 & 19,35 & 7 & 22,58 & \multirow{3}{*}{0,57} \\
\hline & Mensual & 1 & 3,23 & 3 & 9,68 & \\
\hline & Semanal & 4 & 12,9 & 10 & 32,23 & \\
\hline \multirow{2}{*}{ Dependencia laboral } & Dependiente & 5 & 16,1 & 15 & 48,4 & \multirow{2}{*}{0,106} \\
\hline & Independiente & 6 & 19,4 & 16 & 35,5 & \\
\hline \multirow{2}{*}{ Autorización de padres } & $\mathrm{Si}$ & 10 & 32,3 & 17 & 54,8 & \multirow{2}{*}{0,553} \\
\hline & No & 1 & 3,2 & 3 & 9,7 & \\
\hline
\end{tabular}

Nota: Instrumento de evaluación elaborado por autores

representa esa celda. Además, se muestran las pruebas de dependencia de chi cuadrado para la interacción de variables en estudio, con el nivel de logro de aprendizaje como variable dependiente. También se obtuvo que, para el caso de tipo de trabajo, no existe una relación significativa $(\mathrm{p}>$ $0,05)$; es decir, que el tipo de trabajo no influye en el rendimiento académico de los alumnos, sea este formal o informal. Además, se definió que la mayor proporción de alumnos tuvo logros previstos en su aprendizaje y trabaja de manera informal. Para la jornada laboral se demostró que no existe una relación entre variables; es decir, que la jornada laboral no influye en el rendimiento académico de los alumnos, teniendo mayor representatividad aquellos que laboran 48 o menos horas semanales, con 51,6 \% del total. La retribución mensual confrontada con el nivel de logro de aprendizaje muestra que aquellos alumnos cuyos sueldos son mayores a 930 soles y tienen un logro de aprendizaje previsto. Distintivos, con un 35,3\% del total. Además, la prueba de dependencia indica que no existe relación significativa entre la retribución mensual y el nivel de logro de aprendizaje. La tabla también muestra que el periodo de pago no guarda relación con el nivel de logro de aprendizaje, ya que aquellos alumnos que reciben sus pagos diarios tienden a tener la misma apreciación académica que aquellos alumnos que cobran un sueldo semanal o mensual. De estos últimos se reportó que el 32,3 \% recibe sueldos semanales y tiene logro previstos de aprendizaje; siendo los más representativos. La dependencia laboral en relación a la característica de trabajo realizado muestra que no guarda una relación significativa $(p>0,05)$ con el logro de aprendizaje esperado; y es que aquellos alumnos que son independientes o dependientes de un establecimiento tienden a tener las mismas calificaciones académicas. Por último, la variable de autorización paterna para laborar indica que no guarda relación con el nivel de logro de aprendizaje; además, el $54,8 \%$ del total mencionó que tiene autorización de sus padres para trabajar y tienden a tener logros de aprendizaje previstos para su desempeño académico como grupo representativo.

\section{DISCUSIÓN}

Abolir el trabajo infantil y adolescente es una propuesta extrema e imposible, porque el ser humano por naturaleza vive para trabajar y trabaja para vivir; mientras que la otra postura de proteger el trabajo infantil y adolescente sí es razonable (Acevedo et al., 2011). Este trabajo que realizan les ayuda a cubrir los gastos necesarios para su bienestar, por lo que se está de acuerdo con la postura proteccionista ya que aquel es necesario para la vida y para la madurez del adolescente quien invierte el dinero ganado en su educación y salud, además de destinarlo también al ahorro (Otálvaro, 2011). Lo central aquí no es tanto que el adolescente trabaje o no, sino que el trabajo que realiza no interfiera con su permanencia, asistencia y acceso a la institución educativa, de igual manera se le debe de brindar el trato adecuado, no vulnerando sus derechos (Zamora y Carpio, 2016). Los hallazgos de este estudio permiten suponer que el trabajo adolescente se muestra de manera positiva en adolescentes que trabajan, y desarrollan 
capacidades para enfrentarse a situaciones de la vida y condiciones laborales, sin dejar de estudiar ni perjudicar su etapa escolar (Cervini, 2005).

La Constitución Política del Perú prevé como jornada máxima de trabajo ocho horas diarias o 48 horas semanales. Sin embargo, se puede establecer por ley, por convenio o decisión unilateral del empleador una jornada menor a la máxima legal. Así mismo, la convención sobre los derechos del niño y adolescente en su Artículo $56^{\circ}$ (Congreso de la República del Perú, 2000) menciona que el trabajo del adolescente entre los doce y catorce años no excederá de cuatro horas diarias ni de veinticuatro horas semanales; y el adolescente, entre los quince y diecisiete años, no excederá de seis horas diarias ni de treinta y seis horas semanales. Los resultados de este artículo muestran que estos valores se superan sobradamente, enfatizándose la necesidad al momento de la búsqueda de trabajo y mejora de ingresos familiares; en su mayor parte con el consentimiento de los padres. Un problema que refleja el nivel de las desigualdades económicas y sociales en Latinoamérica (Del Rio y Cumsille, 2008).

De acuerdo al enfoque por competencias de la UNESCO, el eje central del aprendizaje se ubica en el logro de capacidades y valores en los estudiantes, a través de contenidos pertinentes y significativos y de metodologías participativas y constructivas del conocimiento y aprendizaje. Los resultados del presente trabajo muestran que no hay un efecto deprimente en el rendimiento académico de los alumnos que trabajan y, aunque es evidente que las estrategias de enseñanza contemporáneas han cambiado radicalmente, hoy en día estas enfatizan el proceso resolutivo de problemas como estrategia de enseñanza y aprendizaje porque los protagonistas principales son los estudiantes y el entorno en el que se desenvuelven (Del Valle y Curotto, 2008). Además, según la normativa peruana, el docente peruano tiene que desarrollar habilidades que implementen actividades secuenciales de carácter interactivo y cooperativo, dinámicas grupales que ayuden a la resolución de problemas por parte de los estudiantes y esto se ve reforzado con situaciones diarias en sus trabajos, apoyando la comunicación de ideas esenciales en la comprensión de un tema o fenómeno determinado, como la presentación de reactivos que fomenten el análisis y la acción (Ocaña, 2009).

A pesar de tener condiciones de trabajo muy básicas y defectuosas con faltas graves a las políticas laborales, los resultados muestran, en definitiva, que los alumnos tienen un buen desempeño académico; lo que denota una por ello una capacidad de autosuperación inmutable que enriquece su formación desde una temprana edad Aunque también se percibe la necesidad de que se protejan los derechos de los niños y adolescentes con normas y condiciones que regulen el trabajo de los adolescentes por parte de los gobiernos locales y nacionales. De acuerdo a los resultados obtenidos en la presente investigación se concluye por lo tanto que el trabajo adolescente no influye significativamente en el rendimiento académico de los estudiantes del CEBA María Inmaculada, ya que el trabajo que realizan durante el día mejora ciertas características de desenvolvimiento, liderazgo y formación en competencias que permiten responder a un mercado de trabajo difícil y cambiante (CEPAL, 2001); además de tener en cuenta la temática y metodología de aprendizaje en la educación peruana mediante aprendizajes focalizados en la resolución de problemas.

\section{REFERENCIAS BIBLIOGRÁFICAS}

Alcázar, L., Rendón, S. y Wachtenheim, E. (2001). Trabajando y estudiando en américa latina rural: decisiones críticas de la adolescencia. Lima, Perú: Instituto Apoyo.

Acevedo, K., Quejada, R., y Yánez, M. (2011). Determinante y consecuencias del trabajo infantil: un análisis de la literatura. Investigación y Reflexión, 19, 113-124.

Comisión Económica para América Latina y el Caribe, CEPAL. (2001). Formación para el trabajo y los jóvenes en América Latina. Santiago de Chile, Chile: CEPAL.

Cervini, R. (2005). Trabajo infantil urbano y logro en matemáticas de la educación básica. Un modelo de dos niveles. Revista Mexicana de Investigación Educativa, 10(25), 451-480.

Chinyoka, K., y Naidu, N. (2014). Underage and Unprotected: Psychosocial Effects of Child Labour on the Academic Performance of Learners in Zimbabwe. Journal of Psychology, 5(1), 65-74. Recuperado de http://doi.org/10.1080/09764224.2014.11885506

Defensoría del Pueblo. (2016). Agenda Legislativa pendiente para la niñez y adolescencia en el Perú (2017-2021) Recuperado de https://www.unicef.org/peru/sites/unicef.org.peru/files/2019-01/Agenda_legislativa_pendiente_por_la_ninez_y_adolescencia_ en_el_Peru_2017-2021.pdf

Del Rio, M. y Cumsille, P. (2008). ¿Necesidad Económica o Preferencias Culturales? La Justificación Parental del Trabajo Infantil en Chile. Psykhe, 17(2), 41-52.

Del Valle, M., y Curotto, M. (2008). La resolución de problemas como estrategia de enseñanza y aprendizaje. Revista Electrónica de Enseñanza de Las Ciencias, 7, 463-479.

Instituto Nacional de Estadística e Informática, INEI. (2002). Visión del Trabajo Infantil y Adolescente en el Perú, 2001.Recuperado de https://www.inei.gob.pe/media/MenuRecursivo/publicaciones_digitales/Est/ Lib0527/Libro.pdf 
Lawshe, C. (1975). a Quantitative Approach To Content Validity. Personnel Psychology, 28(4), 563-575

Ministerio de Educación, MINEDU. (2019). Resolución Viceministerial ( $\mathrm{N}^{\circ}$ 025-2019). Recuperado de https:// www.gob.pe/institucion/minedu/normas-legales/259702-025-2019-minedu

Ocaña, Y. (2009). Las Ciencias Sociales y su enseñanza: formemos estudiantes creativos, críticos y cooperativos. Investigación Educativa, 13(24), 179-185.

OIT. (2002). Visión del Trabajo Infantil y Adolescente en el Perú, 2001. Recuperado de https://www.inei.gob pe/media/MenuRecursivo/publicaciones_digitales/Est/Lib0527/Libro.pdf

Otálvaro, J. (2011). La crianza del niño trabajador: una reflexión desde la salud pública. Revista Facultad Nacional de Salud Pública, 29(4), 495-503.

Ovalle, J. (2009). Deserción escolar y trabajo adolescente en los beneficiarios de la beca de apoyo a la retención escolar (tesis). Universidad academia de Humanismo Cristiano,Santiago de Chile, Chile.

UNICEF. (2010). Trabajo infantil y niñez indigena en América Latina. Recuperado de http://www.lo.org/wcmsp5/groups/public/---ed_norm/---normes/documents/publication/wcms_150598.pdf

UNICEF. (2016). Agenda Legislativa pendiente para la niñez y adolescencia en el Perú (2017 - 2021). Lima, Perú: UNICEF.
Vilcarana, M. y Cayllahua, P. (2017). Influencia del trabajo que realizan los adolescentes en el rendimiento escolar del centro de educación básica alternativa "Alberto Flores Galindo" Orcopampa, Castilla, Arequipa, 2017 (tesis pregrado). Universidad Nacional de San Agustín de Arequipa, Arequipa, Perú.

Zamora, D. y Carpio, P. (2016). Trabajo infantil y desarrollo, algunos dilemas. En $21^{\circ}$ Encuentro Nacional sobre Desarrollo Regional en México.Mérida,Yucatán. México.

\section{Contribución de los autores}

FMAA: diseño del estudio, recogida de datos, análisis e interpretación de datos, redacto el contenido del artículo. JAL: revisión crítica de su contenido.

CGAS: en la asesoría del trabajo de investigación, revisión y aprobación final de la versión que va a publicarse, redacción y revisión del contenido del artículo.

\section{Fuentes de financiamiento.}

Universidad Nacional del Centro del Perú.

\section{Conflictos de interés}

\section{Ninguno.}

\section{Correspondencia}

Correo: floraraucoacosta31@gmail.com 